\title{
Comments on "A Parameterization of Scale-Dependent Radiative Damping Rates in the Middle Atmosphere"
}

\author{
XUN ZHU \\ Department of Atmospheric Sciences, AK-40, University of Washington, Seattle, WA 98195
}

7 July 1986 and 8 June 1987

Fels (1982, hereafter Fels) presented an analysis of the scale-dependent Newtonian cooling coefficient in the middle atmosphere. The results have been used in some dynamical models (e.g., Dunkerton, 1985; Fels, 1986). It is expected that, like Dickinson's (1973) parameterization, the results will be widely used in the future because of their simple analytical expressions. Fels showed that scale-dependent effects substantially reduced damping times for internal gravity waves with vertical wavelengths less than $15 \mathrm{~km}$. However, he made an assumption in his derivation that the damping time is long compared to the local wave period. This restriction excludes many important waves in the middle atmosphere, e.g., equatorial waves. The purpose of this commentary is to point out that (i) this assumption [(6a) in Fels] can be removed without changing the radiative damping rate, (ii) the results can be extended to planetary waves, and (iii) damping effects will increase the vertical wavelength significantly if the damping rate is comparable to the wave frequency.

For convenience we can neglect the shear effect of the mean flow and derive the following vertical structure equations for $\mathrm{X}$, the $e^{-z / 2 H}$ factored vertical velocity and temperature perturbations in a hydrostatic atmosphere [see $(4 a, b)$ in Fels]:

$$
\begin{aligned}
& \frac{d^{2} \mathrm{X}}{d z^{2}}+n_{0}^{2} \mathrm{X}=\left(n_{0}^{2}+\frac{1}{4 H^{2}}\right) \mu^{-1} \Gamma(T), \\
& -i \omega T+\mu \mathrm{X}=\Gamma(T),
\end{aligned}
$$

where $n_{0}$ is the vertical wavenumber as damping vanishes, e.g. Holton (1979).

For $f$-plane inertio-gravity waves:

$$
n_{0}^{2}=\frac{K^{2} N^{2}}{\omega^{2}-f_{0}^{2}}-\frac{1}{4 H^{2}}
$$

For $\beta$-plane Rossby waves:

$$
n_{0}^{2}=\frac{N^{2}}{f_{0}^{2}}\left(\frac{k \beta}{-\omega}-K^{2}\right)-\frac{1}{4 H^{2}}
$$

For equatorial Kelvin waves:

$$
n_{0}^{2}=\frac{N^{2} k^{2}}{\omega^{2}}-\frac{1}{4 H^{2}}
$$

For mixed Rossby-gravity waves:

$$
n_{0}^{2}=\frac{N^{2} k^{2}}{\omega^{2}}\left(1+\frac{\beta}{\omega k}\right)-\frac{1}{4 H^{2}} .
$$

Here $\omega$ is the Doppler-shifted frequency, $\mu=N^{2} H R^{-1}$, $K^{2}=k^{2}+l^{2}$, and the perturbation radiative cooling term is given by

$$
\Gamma(T)=e^{-z / 2 H} \int_{0}^{\infty} K\left(z, z^{\prime}\right) T\left(z^{\prime}\right) e^{z^{\prime} / 2 H} d z^{\prime} .
$$

For later use in comparison we first solve (1) by the WKB method with a local Newtonian cooling radiative term:

$$
\Gamma(T)=-\alpha_{N} T,
$$

where $\alpha_{N}$ is the Newtonian cooling coefficient. Substituting (4) into (1) and canceling $T$ gives

$$
\frac{d^{2} \mathrm{X}}{d z^{2}}+n^{2} \mathrm{X}=0
$$

in which the revised complex vertical wavenumber $n$ is determined by

$n^{2} \equiv n_{r}^{2}+i n_{i}^{2}=\frac{n_{0}^{2}-(\gamma / 2 H)^{2}}{1+\gamma^{2}}+i \frac{-\gamma\left[n_{0}^{2}+\left(1 / 4 H^{2}\right)\right]}{1+\gamma^{2}}$

where $\gamma=\alpha_{N} / \omega$ is the ratio of the damping rate to the Doppler-shifted frequency. Solving (5) following the conventional WKB method (Wait, 1981) and by substituting $\mathrm{X}(z)=A(z) e^{i \theta(z)}$ into Eq. (5) gives real and imaginary parts:

$$
\begin{aligned}
A_{z z}-A \theta_{z}{ }^{2}+n_{r}{ }^{2} A & =0 . \\
2 A_{z} \theta_{z}+A \theta_{z z}+n_{i}^{2} A & =0 .
\end{aligned}
$$

The slowly varying assumption $\left|A_{z z} / A\right| \ll n_{r}^{2}$ results in the explicit solution of (7): 


$$
\begin{gathered}
\theta_{z}^{2}=n_{r}^{2}=\frac{n_{0}^{2}-(\gamma / 2 H)^{2}}{1+\gamma^{2}} \\
A(z)=A_{0} n_{r}(z)^{-1 / 2} \exp \left(-\int_{0}^{z} \frac{n_{i}\left(z^{\prime}\right)^{2}}{2 n_{r}\left(z^{\prime}\right)} d z^{\prime}\right) .
\end{gathered}
$$

Equation (8a) shows that the damping effect may increase the vertical wavelength significantly if $|\gamma| \geqslant 1$. In the atmosphere, all waves with sources located at lower levels have phase lines tilted downstream of the horizontal phase speed with height. This results in a downward propagation of the wave phase, i.e., $\omega / n_{r}$ $<0$, which makes the wave amplitude decay exponentially with height. Explicit solution (8) derived from WKB approximation fails when the condition $\left|A_{z z}\right| A \mid$ $\ll n_{r}{ }^{2}$ is not satisfied. In that case we must solve (7) for $A$ and $\theta$ simultaneously.

In order to solve the integro-differential equations $(1 \mathrm{a}, \mathrm{b})$ we only require that the kernal function $K(z$, $z^{\prime}$ ) in (3) is sharply peaked around $z^{\prime}=z$ so that for any slowly varying function $A(z)$

$$
\int_{0}^{\infty} K\left(z, z^{\prime}\right) A\left(z^{\prime}\right) e^{i n z^{\prime}} d z^{\prime} \approx A(z) \int_{0}^{\infty} K\left(z, z^{\prime}\right) e^{i n z^{\prime}} d z^{\prime}
$$

This condition is similar to the assumption (b) in Fels. We will see that assumption (c) in Fels, which is equivalent to $\partial K / \partial z \approx-\partial K / \partial z^{\prime}$, is also unnecessary in the following derivations.

With the previously defined condition we can eliminate $T$ in Eq. (1a, b) and show that

$$
\frac{d^{2} \mathrm{X}}{d z^{2}}+n_{0}^{2} \mathrm{X}=i \omega^{-1} \int_{0}^{\infty} K\left(z, z^{\prime}\right)\left(\frac{d^{2} \mathrm{X}\left(z^{\prime}\right)}{d z^{\prime 2}}-\frac{\mathrm{X}\left(z^{\prime}\right)}{4 H^{2}}\right) d z^{\prime}
$$

Similar procedures give the real and imaginary parts of the WKB solution

$$
\begin{aligned}
&\left(1+\frac{\alpha_{i}}{\omega}\right)\left(A_{z z}-A \theta_{z}{ }^{2}\right)+\left(n_{0}{ }^{2}-\frac{\alpha_{i}}{4 H^{2} \omega}\right) A \\
&+\frac{\alpha_{r}}{\omega}\left(2 A_{z} \theta_{z}+A \theta_{z z}\right)=0, \\
&\left(1+\frac{\alpha_{i}}{\omega}\right)\left(2 A_{z} \theta_{z}+A \theta_{z z}\right)-\frac{\alpha_{r}}{\omega}\left(A_{z z}-A \theta_{z}{ }^{2}-\frac{A}{4 H^{2}}\right)=0,
\end{aligned}
$$

where

$$
\begin{aligned}
& \alpha_{r}=\int_{0}^{\infty} K\left(z, z^{\prime}\right) \cos \left[\theta\left(z^{\prime}\right)-\theta(z)\right] d z^{\prime} \\
& \alpha_{i}=\int_{0}^{\infty} K\left(z, z^{\prime}\right) \sin \left[\theta\left(z^{\prime}\right)-\theta(z)\right] d z^{\prime}
\end{aligned}
$$

We can rewrite (11) in the form of (7) by solving it for $\left(A_{z z}-A \theta_{z}^{2}\right)$ and $\left(2 A_{z} \theta_{z}+A \theta_{z z}\right)$. Further simplification can be made with the condition that $\left|\alpha_{i} / \omega\right| \ll 1$, which is a very good approximation for all waves in the real atmosphere. We then have

$$
\begin{aligned}
& \left(1+\gamma_{r}{ }^{2}\right)\left(A_{z z}-A \theta_{z}{ }^{2}\right)+\left[n_{0}{ }^{2}-\left(\frac{\gamma_{r}}{2 H}\right)^{2}\right] A=0, \\
& \left(1+\gamma_{r}^{2}\right)\left(2 A_{z} \theta_{z}+A \theta_{z z}\right)-\gamma_{r}\left(n_{0}^{2}+\frac{1}{4 H^{2}}\right) A=0,
\end{aligned}
$$

where $\gamma_{r}=-\alpha_{r} / \omega$. Comparing (13) to (7) immediately shows that the scale-dependent generalization of Newtonian cooling is given by

$\tau^{-1}(n, z)=-\alpha_{r}=-\int_{0}^{\infty} K\left(z, z^{\prime}\right) \cos \left[\theta\left(z^{\prime}\right)-\theta(z)\right] d z^{\prime}$

The result is the same as Fels' (a minus sign was left out in his paper).

In view of this analysis we see that Fels' results can be used for all waves in the middle atmosphere, no matter what their periods and wavelengths are. The vertical wavelength may be increased by radiative damping. However, such an effect is mainly due to $\alpha_{r}$ but not to $\alpha_{i}$.

Acknowledgments. The author wishes to thank Dr. Conway B. Leovy for his helpful suggestions. This work was supported by the National Aeronautics and Space Administrations through Grant NAGW-662.

\section{REFERENCES}

Dunkerton, T. J., 1985: A two-dimensional model of the quasi-biennial oscillation. J. Atmos. Sci., 42, 1151-1160.

Fels, S. B., 1982: A parameterization of scale-dependent radiative damping rates in the middle atmosphere. J. Atmos. Sci., 39, 1141-1152.

—_, 1986: Radiative-dynamical interactions in the middle atmosphere. Adv. Geophys., 28A, 277-300.

Holton, J. R., 1979: An Introduction to the Dynamic Meteorology. Academic Press.

Wait, J. R., 1981: Wave Propagation Theory. Pergamon Press. 